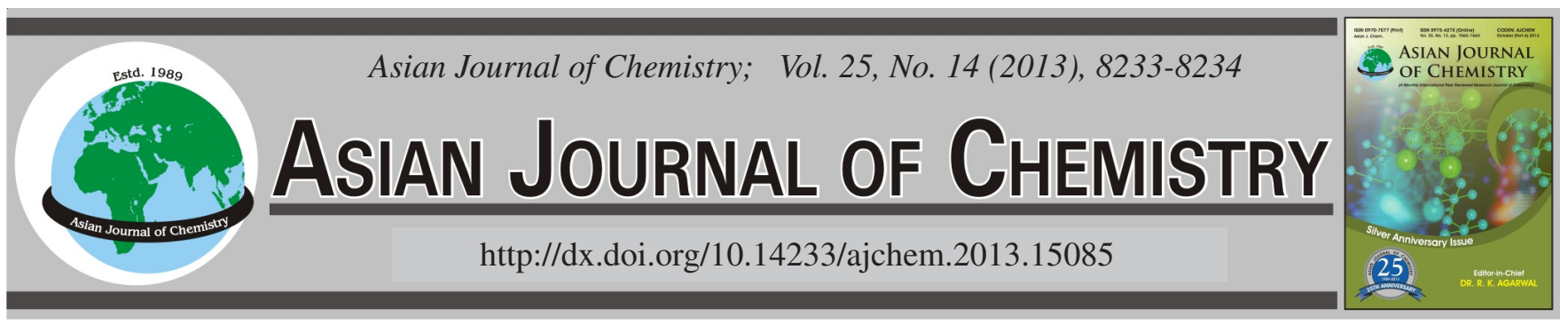

NOTE

\title{
Hydrothermal Synthesis and Crystal Structure of A New 1D Chain Mn(II) Coordination Polymer
}

\section{Bo SHEN and XIANJIANG $\mathrm{LI}^{*}$}

Shandong Shengli Co. Ltd., Jinan 250101, Shandong Province, P.R. China

*Corresponding author: E-mail: whyull@yeah.net

\begin{abstract}
One new $\mathrm{Mn}(\mathrm{II})$ compound $\left\{\left[\mathrm{Mn}(\mathrm{L})\left(\mathrm{H}_{2} \mathrm{O}\right)\right] \cdot 2 \mathrm{H}_{2} \mathrm{O}\right\}_{n}\left(\mathrm{H}_{2} \mathrm{~L}=5\right.$-methylisophthalic acid $)$ has been successfully synthesized. Compound shows a one-dimensional chain. The carboxylate group in compound adopt two coordination modes viz., bis-chelating and bis-monodentate modes.
\end{abstract}

Key Words: Coordination polymer, Crystal structure, Mn(II).

The rational design and construction of novel functional metal-organic frameworks (MOFs) is currently of great interest for the past decade due to their diverse topologies and potential applications in gas storage, magnetism, catalysis and luminescence ${ }^{1-4}$. Although the rapid progress in metal-organic frameworks has been made, it is also a great challenge to rationally prepare and control the structures and composition of target products in crystal engineering because of the difficult prediction of either the composition or the structure of the reaction product.

All reagent and solvents employed were commercially available and used as received without further purification.

Preparation of compound: A mixture of 5-methylisophthalic acid (1 mmol), $\mathrm{Mn}(\mathrm{OAc})_{2} \cdot 2 \mathrm{H}_{2} \mathrm{O}$ (1 mmol), bis $(1$ mmol) and distilled water $(15 \mathrm{~mL})$ was heated in a $25 \mathrm{~mL}$ stainless steel reactor with a Teflon liner $160^{\circ} \mathrm{C}$ for $96 \mathrm{~h}$, followed by slow cooling to room temperature. Yellow crystals of the compound formed (Fig. 1).

X-Crystallography: Suitable single crystals were selected under a polarizing microscope and fixed with epoxy cement on fine glass fibers which were mounted on a Bruker Smart

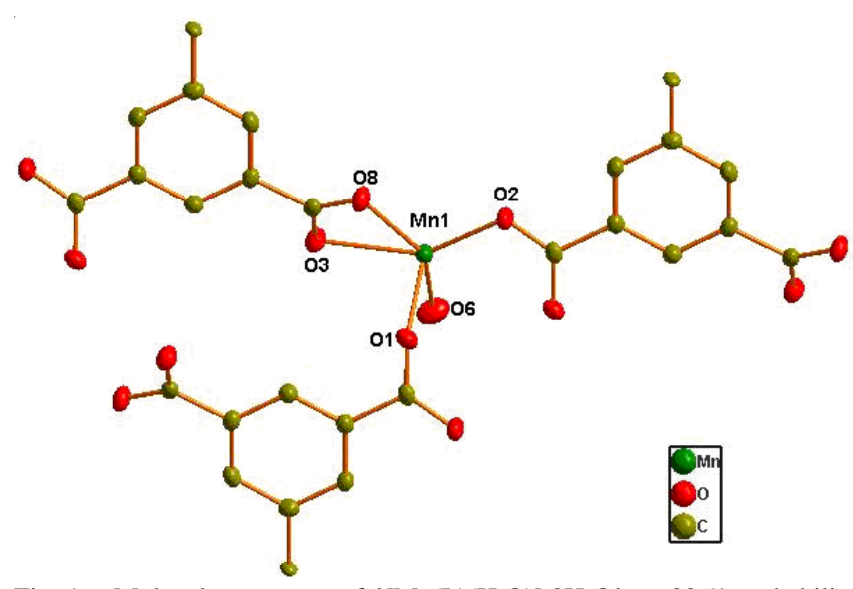

Fig. 1. Molecular structure of $\left\{\left[\mathrm{Mn}(\mathrm{L})\left(\mathrm{H}_{2} \mathrm{O}\right)\right] \cdot 2 \mathrm{H}_{2} \mathrm{O}\right\}_{\mathrm{n}}$ at $30 \%$ probability displacement ellipsoids

$1000 \mathrm{CCD}$ diffractometer with a $\mathrm{MoK}_{\alpha}$ radiation $(\lambda=0.71073$ $\AA$ ) at 293(2) K. The hydrogen atoms bound to carbon were located by geometrically calculations. All non-hydrogen atoms were refined by full-matrix least-squares techniques. All calculations were performed by the SHELXTL 97 program $^{5}$. The

\begin{tabular}{|c|c|c|c|}
\hline \multicolumn{4}{|c|}{$\begin{array}{l}\text { TABLE-1 } \\
\text { JCTURE RE }\end{array}$} \\
\hline Empirical formula & $\mathrm{C}_{9} \mathrm{H}_{11} \mathrm{O}_{7} \mathrm{Mn}$ & $\mathrm{Z}$, Calculated density $\left(\mathrm{mg} / \mathrm{m}^{3}\right)$ & $2,1.839$ \\
\hline Formula weight & 286.12 & Absorption coefficient $\left(\mathrm{mm}^{-1}\right)$ & 1.299 \\
\hline Crystal system space group & Triclinic, P-1 & $F_{(000)}$ & 292 \\
\hline Unit cell dimensions & $\begin{array}{c}\mathrm{a}=7.752(5) \AA ⿻ \mathrm{\AA}, \mathrm{b}=8.606(5) \\
\AA, \mathrm{c}=8.702(5) \AA\end{array}$ & Limiting indices & $-6 \leq \mathrm{h} \leq 9,-6 \leq \mathrm{k} \leq 10,-10 \leq 1 \leq 10$ \\
\hline Volume $\left(\AA^{3}\right)$ & $516.8(2)$ & Largest diff. peak and hole $\left(\mathrm{e} / \AA^{3}\right)$ & 0.841 and -0.491 \\
\hline$\theta$ range for data collection & $2.41-25.00$ & Goodness-of-fit on $\mathrm{F}^{2}$ & 1.090 \\
\hline Final $\mathrm{R}$ indices $[\mathrm{I}>2 \sigma(\mathrm{I})]$ & $\mathrm{R}_{1}=0.0341 \mathrm{wR}_{2}=0.1060$ & $\mathrm{R}$ indices (all data) & $\mathrm{R}_{1}=0.0348, \mathrm{wR}_{2}=0.1073$ \\
\hline
\end{tabular}




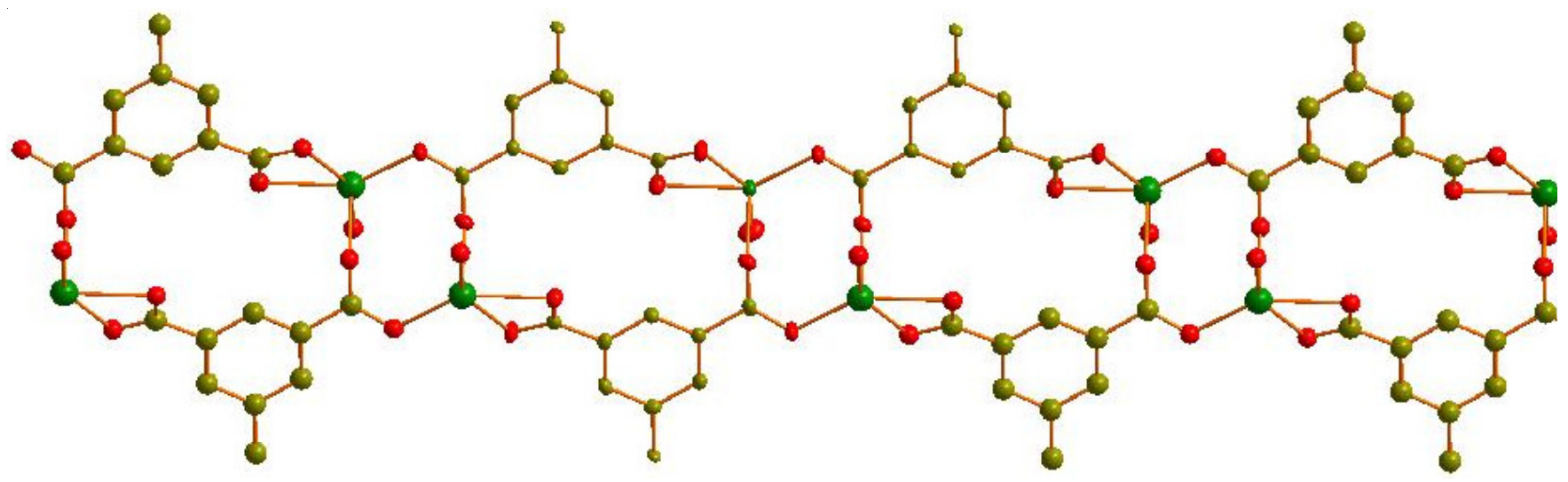

Fig. 2

crystallographic data and experimental details of structural analyses for coordination polymers are summarized in Table-1. Selected bond and angle parameters are listed in Table- 2 .

\begin{tabular}{|c|c|c|c|}
\hline \multicolumn{4}{|c|}{$\begin{array}{l}\text { TABLE-2 } \\
\text { SELECTED BOND LENGTHS }(\AA) \\
\text { AND ANGLES }\left({ }^{\circ}\right) \text { FOR COMPLEX }\end{array}$} \\
\hline $\mathrm{Cd} 1-\mathrm{O} 4^{\mathrm{i}}$ & $2.2416(14)$ & $\mathrm{Cd} 1-\mathrm{O} 1^{\mathrm{iii}}$ & $2.3117(14)$ \\
\hline $\mathrm{Cd} 1-\mathrm{O} 4^{\mathrm{ii}}$ & 2.2416 (14) & $\mathrm{Cd} 1-\mathrm{O} 2^{\mathrm{iii}}$ & $2.5038(14)$ \\
\hline Cd1-O1a & $2.3117(14)$ & $\mathrm{Cd} 1-\mathrm{O} 2 \mathrm{a}$ & $2.5038(14)$ \\
\hline $\mathrm{O} 4^{\mathrm{i}}-\mathrm{Cd} 1-\mathrm{O} 4^{\mathrm{ii}}$ & $102.69(8)$ & $\mathrm{O} 4^{\mathrm{ii}}-\mathrm{Cd} 1-\mathrm{O} 1^{\mathrm{iii}}$ & $127.85(5)$ \\
\hline $\mathrm{O} 4$-Cd1-O1a & $127.85(5)$ & $\mathrm{O} 1 \mathrm{a}-\mathrm{Cd} 1-\mathrm{O} 1^{\mathrm{iii}}$ & $125.85(7)$ \\
\hline $\mathrm{O} 4^{\mathrm{ii}-\mathrm{Cd} 1-\mathrm{O} 1 \mathrm{a}}$ & $87.42(5)$ & $\mathrm{O} 4^{\mathrm{i}}-\mathrm{Cd} 1-\mathrm{O} 2^{\mathrm{iii}}$ & $139.16(5)$ \\
\hline 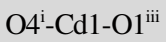 & $87.42(5)$ & $\mathrm{O} 4^{\mathrm{ii}}-\mathrm{Cd} 1-\mathrm{O} 2^{\mathrm{iii}}$ & $92.44(5)$ \\
\hline
\end{tabular}

Symmetry codes: (i) $-\mathrm{x}+1 / 2, \mathrm{y}+1 / 2,-\mathrm{z}+1 / 2$; (ii) $\mathrm{x}-1 / 2, \mathrm{y}+1 / 2$, $\mathrm{z}$; (iii) $-\mathrm{x}$, $\mathrm{y},-\mathrm{z}+1 / 2$.
Structure description: $\operatorname{In}\left\{\left[\mathrm{Mn}(\mathrm{L})\left(\mathrm{H}_{2} \mathrm{O}\right)\right] \cdot 2 \mathrm{H}_{2} \mathrm{O}\right\}_{n}, \mathrm{Mn}(\mathrm{II})$ atom is coordinated to five oxygen atoms from three $\mathrm{L}$ ligands and one oxygen atom from coordination water molecule. In compound the carboxylate groups adopt two coordinated modes: bis-chelating and bis-monodentate modes, which further connect the Mn atoms to form a one-dimensional chains (Fig. 2).

\section{REFERENCES}

1. M.D. Ward, Cood. Chem. Rev. 251, 1663 (2007).

2. Q. Chen, J.B. Lin, W.Xue, M.H. Zeng and X.M. Chen, Inorg. Chem., 50, 2321 (2011).

3. R.Q. Zou, R.Q. Zhong, M. Du, D.S. Pandey and Q. Xu, Cryst. Growth Des., 8, 452 (2008).

4. Y. Wu, B.-H. Yang, M. Li, C.-A. Tian and L.-G. Qiu, Asian J. Chem., 24, 4044 (2012).

5. G.M. Sheldrick, SHELXTL97, Program for the Refinement of Crystal Structure, University of Gottingen, Germany (1997). 